\section{Comparison of retinal nerve fibre layers between 11778 and 14484 mutations in Leber's hereditary optic neuropathy}

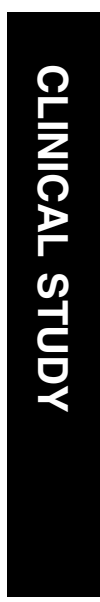

${ }^{1}$ Department of Ophthalmology, Seoul National University College of Medicine, Seoul, Korea

${ }^{2}$ Seoul Artificial Eye Center, Seoul National University Hospital Clinical Research Institute, Seoul, Korea

${ }^{3}$ Department of Ophthalmology, Seoul National University Bundang Hospital, Seongnam, Korea

${ }^{4}$ Department of laboratory medicine, Seoul National University College of Medicine, Seoul, Korea

Correspondence: J-M Hwang, Department of Ophthalmology, Seoul National University Bundang Hospital, 300 Gumi-dong, Bundang-gu, Seongnam, Gyeonggi-do 463-707, Korea

Tel: + 82317877372 ;

Fax: +82 317876838

E-mail: hjm@

snu.ac.kr

Received: 25 September 2008

Accepted in revised form: 22 January 2009

Published online: 27

February 2009

The authors have no vested interest of any kind in the materials or services referred to in this article. optical coherence tomography; retinal nerve patients with the 11778 mutation and 7 patients with the 14484 mutation were enrolled. There was no unilaterally affected case in this study. 
Patients were divided into four groups according to disease duration, that is, $\leqslant 6$ months or $>6$ months, ${ }^{7}$ and according to mtDNA mutation type. These four groups were referred to as early 11778 , late 11778 , early 14484 , and late 14484 groups. Nine eyes of the early 11778 group repeatedly underwent OCT and were also included in the late 11778 group.

\section{Methods}

All of the patients underwent a thorough relevant history taking (onset of disease, family history, medication, earlier affected disease, disease duration, onset age, visual recovery, etc) and ophthalmological examinations including the measurement of best-corrected visual acuity, pupillary examination, colour vision test of Ishihara and the Hardy-Rand-Rittler test, direct and indirect ophthalmoscopy, fundus photography, Goldmann or Humphrey visual field test, and OCT. RNFL thicknesses were measured using a Stratus OCT (Carl Zeiss Inc., Dublin, CA, USA), a low-coherence interferometry unit that generates a cross-sectional image. A fast RNFL thickness scan acquisition protocol, which acquired and compressed three $3.4 \mathrm{~mm}$ RNFL thickness circle scans into one scan, was used for the RNFL analysis. Pupillary dilation was induced in all patients, and internal fixation was used whenever possible. Repeated measurements were taken by an expert technician, and only those images with good fixations and signal strength greater than 6 were selected. Average RNFL thickness, and 4-quadrant RNFL thickness were determined for each eye.

We defined visual recovery as an improvement of visual acuity $>+0.7 \log$ MAR or improvements in visual field such as the reduction of central scotoma, and evaluated recovery rate according to the mtDNA type. Our Institutional Review Board approved the study protocol, informed consent was obtained from all of the subjects, and all procedures used were consistent with the tenets of the Helsinki Declaration.

\section{Statistical analysis}

Age distributions, percentages of males, disease durations, ages at onset, visual recovery rates, and average RNFL thickness were compared with respect to the 11778 and 14484 mutations using the $\chi^{2}$ test and the independent $t$-test. RNFL thickness was compared between the early 11778 and the early 14484 groups, and between the late 11778 and the late 14484 groups using the independent $t$-test, and if the number of cases was $<30$, additional analysis was performed using the Mann-Whitney $U$ test. Statistical analyses were performed using SPSS v.12.0 software (SPSS Inc., Chicago, IL, USA), and two-sided $P$-values of $<0.05$ were considered statistically significant.

\section{Results}

The demographic and ophthalmic data of the 30 patients enrolled in this study are presented in Table 1. Twentythree patients (46 eyes) with the 11778 mutation and seven patients (14 eyes) with the 14484 mutation were enrolled. There were only two patients in a same pedigree in 14484 group (Cases no. 2 and 4). In 11778 group, 15 eyes of 46 eyes were early LHON and 31 eyes were late LHON. Whereas, in 14484 group, four eyes of

Table 1 Demographics and clinical characteristics of the enrolled patients

\begin{tabular}{lccc}
\hline & $11778(\mathrm{~N}=46$ eyes $)$ & $14484(\mathrm{~N}=14$ eyes $)$ & P-value \\
\hline Age (years) & $25.5 \pm 9.7$ & $24.4 \pm 7.2$ & $0.69^{\mathrm{a}}$ \\
Gender, male $n(\%)$ & $42(91.3 \%)$ & $13(92.9 \%)$ & $0.55^{\mathrm{a}}$ \\
Disease duration (years) & $4.0 \pm 5.3$ & $4.8 \pm 6.1$ & $0.61^{\mathrm{a}}$ \\
Age of onset (years) & $21.5 \pm 9.0$ & $19.6 \pm 7.9$ & $0.47^{\mathrm{a}}$ \\
Initial visual acuity ${ }^{\mathrm{b}}(\operatorname{logMAR})$ & $1.09 \pm 0.57$ & $0.95 \pm 0.44$ & 0.38 \\
Final visual acuity ${ }^{\mathrm{b}}(\operatorname{logMAR})$ & $1.16 \pm 0.55$ & $0.41 \pm 0.62$ & 0.0001 \\
Early LHON, $N(\%)$ & $15(32.6 \%)$ & $10(71.4 \%)$ & $0.78^{\mathrm{a}}$ \\
Atrophy LHON, $N(\%)$ & $31(67.4 \%)$ & $10(71.4 \%)$ & $0.78^{\mathrm{a}}$ \\
Visual recovery, $N(\%)$ & $13(28.3 \%)$ & & $0.003^{\mathrm{a}}$ \\
$R N F L$ thickness ${ }^{b}(\mu m)$ & & $92.9 \pm 23.6$ & 0.36 \\
$\quad$ Average & $82.4 \pm 35.7$ & $123.5 \pm 35.1$ & $75.5 \pm 15.7$ \\
$\quad$ Temporal & $53.6 \pm 31.8$ & $119.5 \pm 31.5$ & 0.66 \\
$\quad$ Superior & $106.7 \pm 49.0$ & & 0.18 \\
$\quad$ Nasal & $66.7 \pm 22.9$ & & 0.08 \\
$\quad$ Inferior & $102.7 \pm 52.0$ & & 0.16 \\
\hline${ }^{a} \chi^{2}$ test. & &
\end{tabular}


Table 2 Retinal nerve fibre layer thickness $(\mu \mathrm{m})$ in Leber's hereditary optic neuropathy patients with a 11778 or 14484 mutation during the early disease stage

\begin{tabular}{lccr}
\hline & $11778(\mathrm{~N}=15 \text { eyes })^{\mathrm{a}}$ & $14484(\mathrm{~N}=4$ eyes $)$ & P-value $^{\mathrm{b}}$ \\
\cline { 2 - 3 } & Mean $\pm S D$ & Mean $\pm S D$ & \\
\hline Average & $130.4 \pm 13.6$ & $117.5 \pm 4.1$ & 0.04 \\
Temporal & $89.9 \pm 32.0$ & $85.8 \pm 18.9$ & 0.96 \\
Superior & $171.9 \pm 17.7$ & $158.7 \pm 10.8$ & 0.15 \\
Nasal & $87.9 \pm 22.6$ & $90.5 \pm 8.4$ & 0.59 \\
Inferior & $171.7 \pm 26.4$ & $135.0 \pm 19.0$ & 0.01 \\
\hline
\end{tabular}

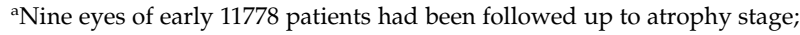
OCT was performed at both early and atrophy stages.

${ }^{\mathrm{b}}$ Mann-Whitney $U$ Test.

Table 3 Retinal nerve fibre layer thickness $(\mu \mathrm{m})$ in Leber's hereditary optic neuropathy patients with a 11778 or 14484 mutation during the late disease stage

\begin{tabular}{lccc}
\hline & $11778(\mathrm{~N}=40 \text { eyes })^{\mathrm{a}}$ & $14484(\mathrm{~N}=10$ eyes $)$ & P-value $^{\mathrm{b}}$ \\
\cline { 2 - 3 } & Mean $\pm S D$ & Mean $\pm S D$ & \\
\hline Average & $65.6 \pm 22.2$ & $81.0 \pm 18.9$ & 0.02 \\
Temporal & $40.5 \pm 17.3$ & $38.2 \pm 7.7$ & 0.73 \\
Superior & $86.2 \pm 35.9$ & $106.5 \pm 28.4$ & 0.023 \\
Nasal & $58.7 \pm 15.8$ & $70.7 \pm 12.3$ & 0.015 \\
Inferior & $76.9 \pm 28.5$ & $108.7 \pm 31.4$ & 0.003 \\
\hline
\end{tabular}

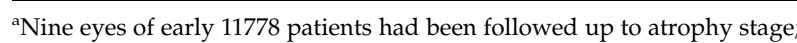
OCT was performed at both early and atrophy stage.

bIndependent $t$-test.

14 eyes were early LHON and 10 eyes were late LHON. For those with the 11778 or 14484 mutation, mean age at onset of LHON (21.5 \pm 9.0 vs $19.6 \pm 7.0$ years, $P=0.47)$, mean disease duration ( $4.0 \pm 5.3$ vs $4.8 \pm 6.1$ years, $P=0.61$ ), and mean age whilst participating in this study (25.5 \pm 9.7 vs $24.4 \pm 7.2$ years, $P=0.69$ ) were similar. Initial visual acuities were not different $(1.09 \pm 0.57$ vs $0.95 \pm 0.44$, in $\log \mathrm{MAR}, P=0.38$ ); however, final visual acuities did differ $(1.16 \pm 0.55$ vs $0.41 \pm 0.62$, in $\log \mathrm{MAR}$, $P=0.0001)$ and visual recovery rate was different (28.3 vs $71.4 \%, P=0.003$ ) between the patients with 11778 or 14484 mutation.

Average RNFL thickness was significantly greater in the early 11778 group than in the early 14484 group (130.4 \pm 14.0 vs $117.5 \pm 4.1 \mu \mathrm{m}, P=0.04$, Table 2 ). Superior quadrant RNFL thickness in the early 11778 and the early 14484 patients were similar $(171.9 \pm 17.7$ vs $158.7 \pm 10.8 \mu \mathrm{m}$, $P=0.15$ ), whereas inferior quadrant RNFL thickness were significantly greater in the early 11778 group than in the early 14484 group $(171.7 \pm 26.4$ vs $135.0 \pm 19.0 \mu \mathrm{m}$, $P=0.01$ ).

RNFL thickness was significantly reduced in the late 11778 group compared with that in the late 14484 group $(65.6 \pm 22.2$ vs $81.0 \pm 18.9 \mu \mathrm{m}, P=0.02$, Table 3$)$. Average superior, nasal, and inferior quadrant RNFL thicknesses in the late 11778 patients were significantly thinner than those in the late 14484 patients $(P=0.023,0.015$, 0.003 , respectively), whereas, average RNFL thicknesses of temporal quadrants were similar in the late 11778 and late 14484 groups $(40.5 \pm 17.3$ vs $38.2 \pm 7.7 \mu \mathrm{m}$, $P=0.73$ ).

Figure 1 showed representative cases of RNFL thickness in early 11778, early 14484, late 11778, and late 14484 groups. RNFL thickness was significantly increased in the early stage (Figure 1a) and decreased in the late stage in the 11778 group than in the 14484 group (Figure 1b).

\section{Discussion}

This is the first study to evaluate RNFL pattern according to the mtDNA mutations of 14484 vs 11778, as well as to the early and late stages. The recently developed third generation OCT, Stratus OCT, has a RNFL resolution of $8-10 \mu \mathrm{m} .{ }^{10,11}$ An earlier study using a Stratus OCT in patients with early LHON showed greatest increases of RNFL thickness in the superior quadrant, followed by the nasal and inferior quadrants, with no significant change in the temporal quadrant. ${ }^{7}$ These findings corresponded with our results, and could be explained by the fact that papillomacular bundle might be the early main target in the early stage of LHON. ${ }^{7,12}$

This study revealed further information on RNFL changes according to mutation type. Our study showed that the superior, nasal, and inferior quadrant of the 11778 group showed more prominent RNFL atrophy than in those of the 14484 group. An additional important finding in this study is that OCT may be helpful in differentiating RNFL edema in LHON and optic neuritis, as the superior and inferior bundles are more affected in LHON, whereas all quadrants are affected in optic neuritis. ${ }^{13-15}$

There are at least two limitations in our study. The number of early 14484 groups was limited. However, the rare incidence of LHON with the $14484 \mathrm{mtDNA}$ mutation should also be considered and the number of the eyes could be analysed with a non-parametric statistical method. In addition, both eyes were compared. However, RNFL thickness was not symmetric at all and the differences were so remarkable between the first and the later affected eyes, especially in the early sages of LHON.

In conclusion, patients with the 11778 mutation showed more severe RNFL edema during early stage disease and more severe RNFL atrophy during the late stage than patients with the 14484 mutation. These findings may be helpful in understanding the different visual prognoses between patients with 11778 vs 14484 mtDNA mutation. 

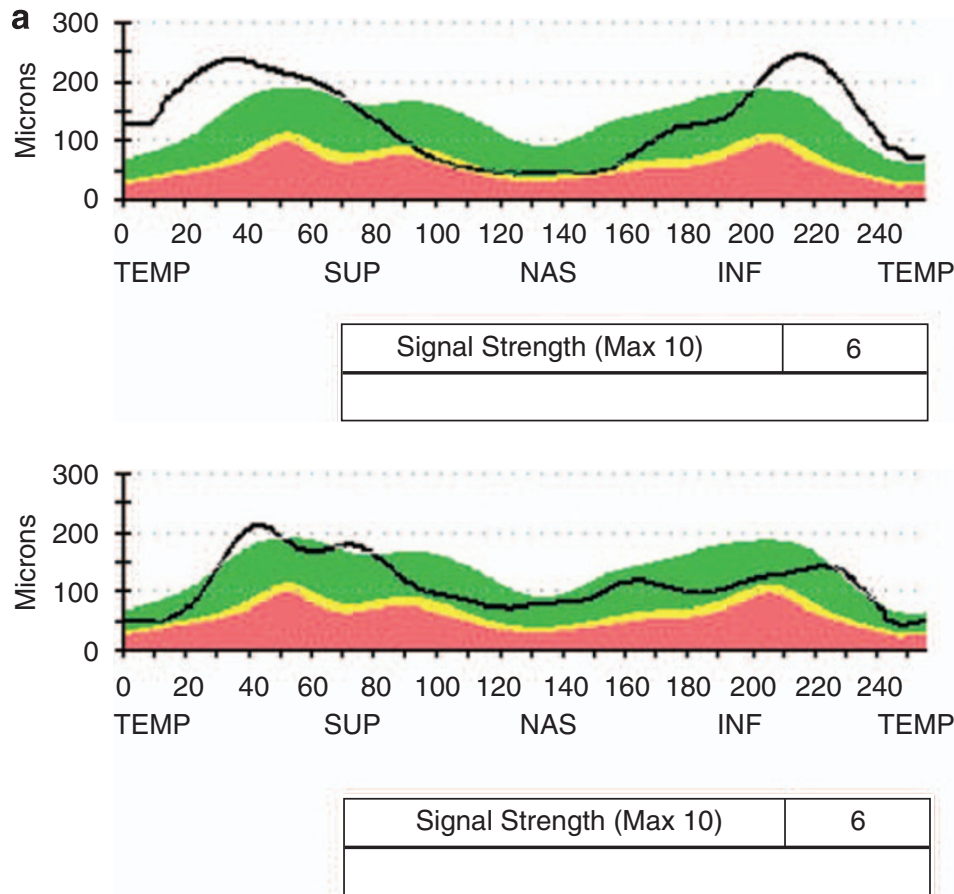

b

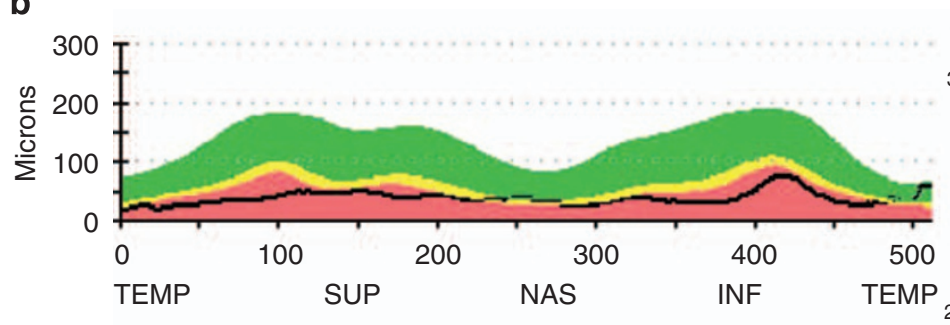

Signal Strength (Max 10)

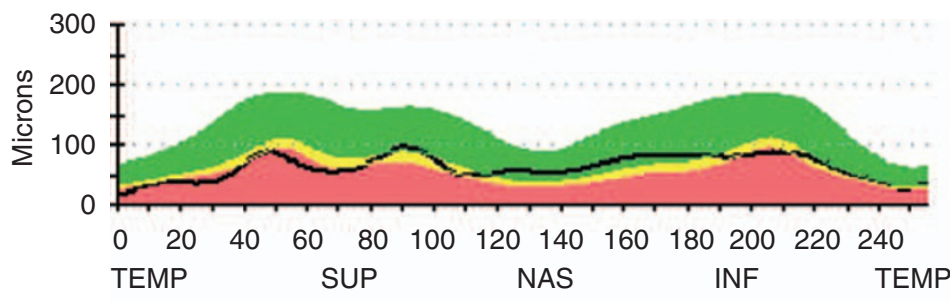

\begin{tabular}{|l|l|}
\hline Signal Strength (Max 10) & 6 \\
\hline
\end{tabular}
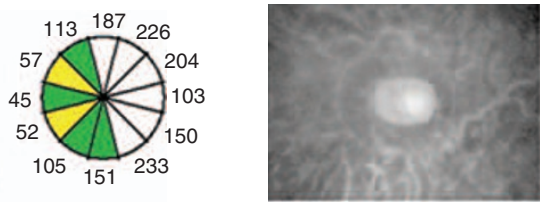

OS
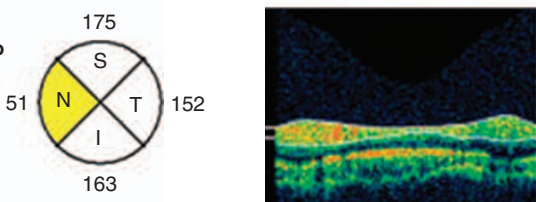

11778
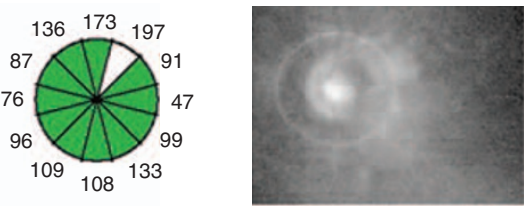

OS

14484
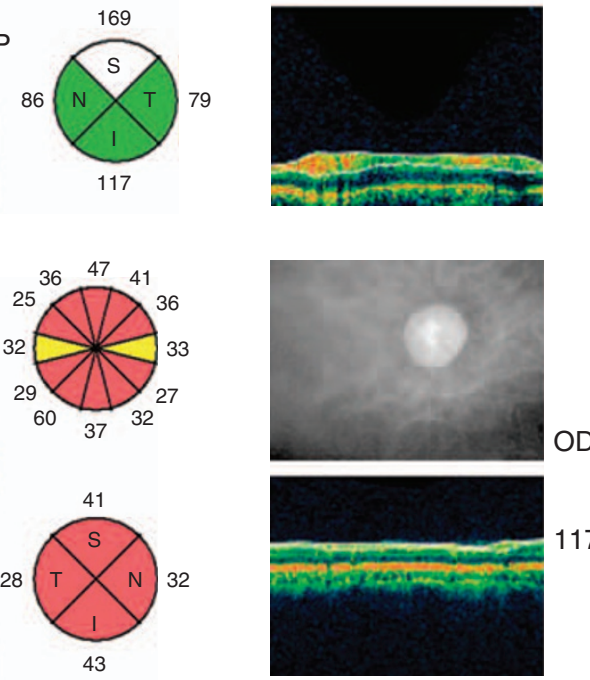

43
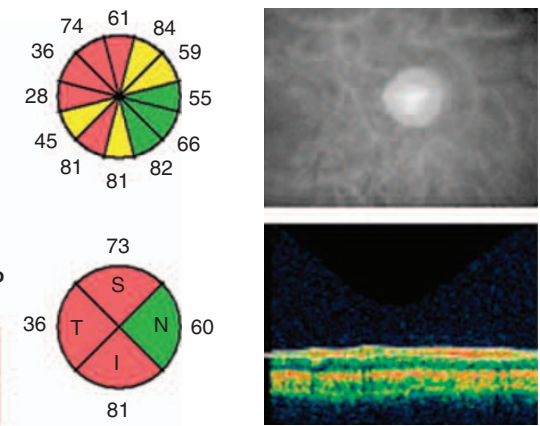

OD

14484

Figure 1 Retinal nerve fibre layer (RNFL) thickness changes in patients with the 11778 or 14484 mutation. (a) RNFL thickness in patients with the 11778 or 14484 mutation during early stage Leber's hereditary optic neuropathy (LHON). The superior quadrant RNFL thickness in both groups showed prominent RNFL edema, whereas average inferior quadrant RNFL thickness was significantly greater in the early 11778 group than in the early 14484 group. (b) RNFL thickness in the late 11778 and 14484 groups. Superior, nasal, and inferior quadrant RNFL thickness in the late 11778 group showed more severe RNFL atrophy than in the late 14484 group. 


\section{Acknowledgements}

This work was supported by the Basic Research Program of the Korean Science \& Engineering Foundation (Grant no. R01-2005-000-10875-0).

\section{References}

1 Newman NJ. Hereditary optic neuropathies. In: Miller NR, Newman NJ (eds). Walsh and Hoyt's Clinical Neuro-ophthalmology, 5th ed. Williams \& Wilkins: Baltimore, 1998, pp 742-753.

2 Nikoskelainen EK, Hoyt WF, Nummelin K. Ophthalmoscopic findings in Leber's hereditary optic neuropathy, I: the fundus findings in asymptomatic family member's. Arch Ophthalmol 1982; 100: 1597-1602.

3 Nikoskelainen EK, Hoyt WF, Nummelin K. Ophthalmoscopic findings in Leber's hereditary optic neuropathy, II: the fundus findings in the affected family member's. Arch Ophthalmol 1983; 101: 1059-1068.

4 Wallace DC, Singh G, Lott MT, Hodge JA, Schurr TG, Lezza AM et al. Mitochondrial DNA mutation associated with Leber's hereditary optic neuropathy. Science 1988; 242: 1427-1430.

5 Mackey D, Howell N. A variant of Leber hereditary optic neuropathy characterized by recovery of vision and by an unusual mitochondrial genetic etiology. Am J Hum Genet 1991; 51: 1218-1228.

6 Huoponen K, Villki J, Aula P, Nikoskelainen EK, Savontaus ML. A new mtDNA mutation associated with Leber hereditary optic neuropathy. Am J Hum Genet 1991; 48 : 1147-1153.
7 Barboni P, Savini G, Valentino ML, Montagna P, Cortelli P, De Negri AM et al. Retinal nerve fiber layer evaluation by optical coherence tomography in Leber's hereditary optic neuropathy. Ophthalmology 2005; 112: 120-126.

8 Savini G, Barboni P, Valentino ML, Montagna P, Cortelli P, De Negri AM et al. Retinal nerve fiber layer evaluation by optical coherence tomography in unaffected carriers with Leber's hereditary optic neuropathy mutations. Ophthalmology 2005; 112: 127-131.

9 Barboni P, Savini G, Valentino ML, La Morgia C, Bellusci C, De Negri AM et al. Leber's hereditary optic neuropathy with childhood onset. Invest Ophthalmol Vis Sci 2006; 47: 5303-5309.

10 Blumenthal EZ, Williams JM, Weinreb RN, Girkin CA, Berry CC, Zangwill LM. Reproducibility of nerve fiber layer thickness measurements by use of optical coherence tomography. Ophthalmology 2000; 107: 2278-2282.

11 Brudenz DL, Michael A, Chang RT, McSoley J, Katz J. Sensitivity and specificity of the Stratus OCT for perimetic glaucoma. Ophthalmology 2005; 112: 3-9.

12 Carelli V, Ross-Cisneros FN, Sadun AA. Mitochondrial dysfunction as a cause of optic neuropathies. Prog Retin Eye Res 2004; 23: 53-89.

13 Savini G, Bellusci C, Carbonelli M, Zanini M, Carelli V, Sadun AA et al. Detection and quantification of retinal nerve fiber layer thickness in optic disc edema using Stratus OCT. Arch Ophthalmol 2006; 124: 1111-1117.

14 Pro MJ, Pons ME, Liebmann JM, Ritch R, Zafar S, Lefton D et al. Imaging of the optic disc and retinal nerve fiber layer in acute optic neuritis. J Neurol Sci 2006; 250: 114-119.

15 Menke MN, Feke GT, Trempe CL. OCT measurements in patients with optic disc edema. Invest Ophthalmol Vis Sci 2005; 46: 3807-3811. 